\title{
Towards inclusive digital television
}

\author{
Mark Springett $\cdot$ Mark Rice $\cdot$ Richard Griffiths
}

Published online: 19 November 2011

(C) Springer-Verlag 2011

Over the last decade, the shift from analogue to digital technology has brought about significant changes to the television landscape. The subsequent emergence of mobile, IPTV and other carriers affords the opportunity to creatively reassess how people interact and engage within this diverging medium. Accessibility services to audio-visual content through sign language, subtitles and audio description have become vital forms of interaction for deaf and hard of hearing, those with low literacy or learning disabilities, and blind and partially sighted people. Improved accessibility affords both humane and economic benefits to the wider society, as access to suitable interactive media implies better quality of life, independence and social inclusion. The payback for technology providers is also considerable, as greater inclusion implies broader market potential for commercial products. Digital television (DTV) potentially affords enhancements in communication, quality of life, safety and support of independent living. Related examples include community television applications in the UK, where the medium has been used to support communication between older citizens in public housing and local authority services [5], as well as social television solutions aimed at improving social connectedness for older people living alone [11].

\section{Springett $(\square)$}

Interaction Design Centre, Middlesex University,

London NW4 4BT, UK

e-mail: m.springett@mdx.ac.uk

\section{Rice}

School of Computing, University of Dundee,

Dundee DD1 4HN, UK

R. Griffiths

School of Computing, Mathematical and Information Sciences, University of Brighton, Brighton BN2 4GJ, UK
Despite this, the dangers of a widening digital divide remain. Ease of use of DTV continues to be a formidable problem-in the purchasing, self-installation of equipment and access of content. Consequentially, there are still a significant number of people who will struggle to adapt to using this technology. Freeman and Lessiter [4] classify these vulnerable groups as those on low incomes and/or with a reduction in one or more ability. In particular, there is strong evidence to suggest that reductions in cognition, sensory and manual dexterity can create significant barriers to the access of related interactive services. This includes the poor design of remote controls, both in terms of their design consistency, tactile feedback and appropriate labelling of buttons. These issues can be further exacerbated by age-related changes to working memory, creating additional 'cognitive load' in the mapping and switching of attention between remote control and TV interfaces [2]. Colour contrast, font type, screen size and distance to screen can all invariably affect text legibility; however, in many instances, DTV lacks support features to readily personalize graphical content. As such, there is an increasing consensus that the self-adaptation of the user interface will help improve interaction barriers for a wider spectrum of users. One notable example is the GUIDE project, ${ }^{1}$ which aims to develop design solutions sensitive to the accessibility needs of users who suffer from combinations of progressive perceptual, motor and cognitive problems.

Some progress has been made in exploring accessible interaction modes and metaphors. For example, Cesar et al. [3] investigated the use of a secondary screen for television interaction. Whilst not explicitly in support of inclusive design, this and other alternative input/output configurations clearly have potential accessibility benefits. Springett

\footnotetext{
${ }^{1}$ http://www.guide-project.eu/.
} 
and Griffiths [12] and others have also investigated the use of alternative inputs, such as gesture. Likewise, the space of utilities for exploiting the medium in support of independent living and quality of life is increasingly being investigated [e.g. 6]. However, to reach market penetration, these applications and services have to deal with the lack of interoperability in broadcasting standards and associated middleware platforms. Indeed, there remains strong political disagreement between policy makers and commercial factions in the move towards greater regulatory intervention. In Europe, despite DTV being foreseen to play a pivotal role in ICT inclusion, a lack of open standards can be contributed by a mixture of internal conflicts in policy, variations in platform interactivity, business models and licensing agreements of software between relevant stakeholders [7]. Stand-alone or fully integrated television units, digital receivers also widely vary in their level of functionality, computational power and interactivity. As such, whilst all receivers are underlined by a common hardware infrastructure, next-generation proprietary systems are technically more versatile to the legacy of units that have been in domestic operation for a number of years. This increasing gap in system performance challenges the extent to which cross modal content provision can be adequately supported. A somewhat problematic issue, given that a substantial number of people with disabilities and those on low income are likely to access digital services through cheaper, low-grade receivers with limited functionality.

Furthermore, the rapid shift in the diversity of DTV technology, particularly in terms of the modality of interaction, namely the inclusion of gesture recognition and other advanced methods of input, (e.g. speech detection) confronts the limited set of accessibility guidelines and recommendations that typically report on using the remote control. As such, questions remain to how these emerging paradigms of interaction might assist, or subsequently hinder barriers of access, given an absence of accessibility research in this area. Drawing reference to the games industry, family-orientated gaming consoles like the Nintendo $\mathrm{Wii}^{\mathrm{TM}}$ are already being measured in early clinical trials to determine their efficacy for stroke patients with motor control impairments [10]. Facilitating therapeutic intervention, this is a good example of how a mainstream technology can foster interest towards more assistive needs, compensating for a lack of affordable alternatives.

The divergence of new media also means there is a clear interest in investigating the social space, in delivering more personalized and portable forms of information, video streaming, applications and Internet usage, sold under the notion of TV anytime, anywhere. Consequentially, backtracking to understand the implications of these changes, in the long term, there is a danger to end up with a more fragmented landscape, with more features and interaction styles, but less regulatory control and accessibility provision for those people most at need.

To address the needs and interests of older and impaired citizens, in-depth research is required into the inclusivity of existing and emerging DTV services, including the methods of interaction associated with the mediated technology. Significantly, both these objectives require design approaches that are in themselves inclusive. For example, examining older age groups, the inherent variability in user characteristics has led to criticism over the notion of designing for the average user [8]. On the one hand, there are many healthy older adults whose user expectations will suitably meet the requirements of those they are designed by [9]. On the other hand, as the incidence of severe disability becomes more prominent with age, equally there will be those who require far greater intervention [1]. Similarly, for visual and hearing impairments, different strategies are required to compensate for varying forms of sensory loss. In each case, this requires time and vested interest by designers and relevant practitioners to understand such human diversity.

This special issue includes five representative articles on design inclusion of digital television and user interaction. Rice and Carmichael present a user requirements study into the design of a social DTV-based application for older adults. Their work focused specifically on the types of issues identified in eliciting ideas with participants limited with technological experience. Hulshof, Pemberton and Griffiths describe a user study in which they evaluated the usability of an electronic programme guide for pre-school children aged between 3 and 4 years old. Conducted with children in both Brazil and the United Kingdom, their work highlights an absence of related DTV literature. By contrast, studies of Miotto et al. and Biswas and Robinson report on more quantitative findings. Using a questionnaire survey, Miotto et al. have examined the potential impact of cognitive training applications on DTV, whilst Biswas and Robinson experimentally compare two types of icon searching using a small sample of able bodied, visually and motor impaired users. Finally, Epelde et al. describe the technical framework for the inclusivity of different input devices and associated interactive modality, built on existing standards for DTV. Prototype examples are given to support their arguments.

Each of these articles demonstrates a need for more appropriate user representation in the development of digital television. Participation of those who represent the outer margins of the user spectrum is a key part of the design process. Across the papers, suggested outcomes are in the form of design principles, technical frameworks, and qualitative and statistical analyses.

The study by Rice and Carmichael, employing brainstorming with paper prototyping techniques to elicit design 
requirements with older adults, draws out a number of strengths and weaknesses in the methods applied. Strong associations are identified to more conventional forms of technology (i.e. the analogue telephone), which is perceived to both impede and influence the creative process. Their analysis explores both the design process and the outcomes of these decisions, suggesting that such limitations require innovation in the methods and interactive tools applied. The results identified a number of related issues in the input control and metaphorical features of a communication application.

Hulshof, Pemberton and Griffiths demonstrate a number of strengths and weaknesses in the development of an electronic programme guide prototype. For example, they highlight advantages of using a one-button mouse over a traditional remote control. Their findings suggest limiting the functionality of the remote control to a single button with clear visual feedback on the secondary display. In particular, they provide practical recommendations for appropriate audio and visual feedback, single screen menu structures, and the inclusion of alternative input devices, such as a touch screen display.

By contrast, addressing the poor design of remote control handsets, Epelde et al. describe the software architecture to customize the type of device and user interface used. Their proposed approach is highlighted through a number of application examples for users with cognitive and visual impairments and more generally elderly users. With the aim to improve the accessibility of DTV services, they utilize the open source 'Universal Remote Console' framework, which allows for a combination of devices, such as PDA and PC tablet or smart phone to act as a secondary control.

Biswas and Robinson evaluated two sets of geometric and graphical icons by exploring the gaze patterns of different set of users to determine the effects of font size, icon spacing and completion time. They report on a number of interaction effects between these set of variables and user groups, both in terms of predicted and actual task completion. However, limitations are reported in the predicted models ability to account for fatigue or a learning effect. Based on these results, they propose a simulator model to predict the design requirements for DTV interfaces. The utility of this model is considerable, given the greater preponderance of progressive perceptual impairments in the user population. The developed model can both facilitate the matching of product specifications to user perceptual capabilities and support the design of adjustability for visual displays and handset features.

Finally, Miotto et al. investigated factors affecting the take-up of specialized cognitive training applications across a large sample of younger and older people in the UK, an unexplored area for DTV applications. Seven cluster groups were established and a range of factors influencing the adoption of cognitive training applications identified. Active well-being, healthy lifestyle and technophilia were perceived as potential drivers, whilst negative attitudes towards TV and general 'unprogressiveness' towards technology as surmountable barriers to their usage. These findings are significant in the sense that such 'brain training' applications may present a new consumer market in DTV interactivity.

\section{References}

1. Carmichael, A.: Older users of digital TV—challenges and opportunities. Consumer Policy Rev. 12(6), 198-203 (2002)

2. Carmichael, A., Rice, M., Sloan, S., Gregor, P.: Digital switchover or digital divide: a prognosis for usable and accessible interactive digital television in the UK. Univers Access Inf Soc 4(4), 400-416 (2006)

3. César, P., Bulterman, C.A., Jansen, A.J.: Usages of the secondary screen in an interactive television environment: control, enrich, share, and transfer television content. In: Tscheligi, M., Obrist, M., Lugmayr, A. (eds.) EuroiTV 2008: changing television environments, LNCS 5066, pp. 168-177. Springer, Heidelberg (2008)

4. Freeman, J., Lessiter, J.: Vulnerable consumers in switchoverwho are they and where do they live? i2 media research ltd, London (2004)

5. Kirklees Borough Council UK: The INtouch kirklees digital TV site: lookinglocal.gov.uk/site/files/Case\%20Study\%20-\%20Kirklees. pdf

6. López-de-Ipiña, D., Blanco, S., Laiseca, X., Díaz-de-Sarralde, I.: ElderCare: an interactive TV-based ambient assisted living platform. In: Chen, L., Nugent, C.D., Biswas, J., Hoey, J. (eds.) Activity Recognition in Pervasive Intelligent Environments, vol. 4, pp. 111-125. Atlantic Press, UK (2011). doi:10.2991/97894-91216-05-3_5

7. Matteucci, N.: Open standards and interoperability in EU digital TV: economics and policy issues. Rev. Econ. Res. Copyr. Issues 5(2), 45-70 (2008)

8. Newell, A.: Extra-ordinary human-computer interaction. In: Edwards, A. (ed.) Extraordinary Human-Computer Interaction, Interfaces for Users with Disabilities, pp. 3-19. Cambridge University Press, New York (1995)

9. Rabbitt, P.M.A., Carmichael, A.R.: Designing communications and information handling systems for disabled and elderly users. In: Snel, J., Cremer, R. (eds.) Work and aging: a European perspective, pp. 173-195. Taylor and Francis, London (1994)

10. Saposnik, G., Teasell, R., Mamdani, M., Hall, J., McIlroy, W., Cheung, D., Thorpe, K.E., Cohen, L.G., Bayley, M.: Effectiveness of virtual reality using Wii gaming technology in stroke rehabilitation. A pilot randomized clinical trial and proof of principle. Stroke 41(7), 1477-1484 (2010)

11. Sokoler, T. Svensson, M.: PresenceRemote: embracing ambiguity in the design of Social TV for senior citizens. In: Tscheligi, M., Obrist, M., Lugmayr, A. (eds.) EuroiTV 2008: Changing Television Environments, LNCS 5066. pp. 158-162 (2008)

12. Springett, M., Griffiths, R.: The Use of Stalking-Horse Evaluation Prototypes for Probing DTV Accessibility Requirements, to Appear in, Interactive Digital Television: Technologies and Applications. IRM press, USA (2007) 